\title{
Early prediction of acute kidney injury by machine learning: Should we add the urine output criterion to improve this new tool?
}

\author{
Cyril Busschots Martins, David De Bels, Patrick M. Honore, Sébastien Redant \\ ICU Department, Centre Hospitalier Universitaire Brugmann-Brugmann University Hospital, Brussels, Belgium
}

Different studies relating to the prediction of the development of acute kidney injury (AKI) by machine learning appear in the literature. A study was realized by Martinez et al. on the early prediction of AKI with a machine learning classification system that relies on vital signs, chief complaints, medical history, and active medical visit and laboratory result to predict the development of AKI Stages 1 and 2 in the next 24 to 72 hours. They include a large population examining altogether 91,258 samples. The performance of their algorithm was characterized by an area under the curve (AUC) up to $0.81(95 \%$ confidence interval 0.80 to 0.82 ). They used the kidney disease improving global outcome (KDIGO) Stage 1 and 2 criterion based only serum creatinine. ${ }^{[1]}$ A second study carried out on the prediction of the development of AKI following cardiac surgery by machine learning performed on 671 patients succeeded in achieving an AUC of 0.843 (95\% CI 0.778-0.899). The criterion used is an increase in serum creatinine according to KDIGO. ${ }^{[2]}$

It is surprising that only serum creatinine was chosen to diagnose AKI. A retrospective study of 1,376 intensive care unit (ICU) patients showed that the AKI incidence was only $20 \%$ using the KDIGO criterion based on serum creatinine alone. This incidence rose up to $38 \%$ when the urine output criterion was used in addition to serum creatinine. For the urine output criterion, the median AKI detection delay was $13 \mathrm{~h}$, while for the serum creatinine criterion, this delay was 24 h. ${ }^{[3]}$ A fourmonth prospective study involving 260 patients showed a $24 \%$ incidence of AKI using the renal injury failure loss of kidney end-stage renal failure (RIFLE) criterion based on serum creatinine, and this incidence increased to $45 \%$ when combining serum creatinine together with the urine output. The delay in AKI diagnosis was estimated to be 1 day when not using the urine criterion. ${ }^{[4]}$ Another prospective study, this time using acute kidney injury network (AKIN) criterion, showed an incidence of AKI of $28 \%$ when using serum creatinine. This incidence rose up to $55 \%$ when using the urine output criterion. ${ }^{[5]}$ In view of these data, the use the urine output criterion does permit to detect more and earlier AKI. We are well aware that, in current practice, it is difficult to monitor urine output in non-ICUs. This makes more difficult the use of urine output for the diagnosis of AKI. Likewise, the urine output is influenced by the body fluid volume of the patient and by the use of diuretics. These reasons explain that many studies have been based solely on the value of blood creatinine. However, adding these diagnostic criteria seems important to us for future investigations in order to improve the performance of these machines learning systems.

\section{Conflict of Interests}

The authors declare that they have no competing interests.

\section{REFERENCES}

1. Martinez DA, Levin SR, Klein EY, Parikh CR, Menez S, Taylor RA, et al. Early Prediction of Acute Kidney Injury in the Emergency Department With Machine- 
Learning Methods Applied to Electronic Health Record Data. Ann Emerg Med 2020; 76: 501-14.

2. Tseng PY, Chen YT, Wang CH, Chiu KM, Peng YS, Hsu SP, et al. Prediction of the development of acute kidney injury following cardiac surgery by machine learning. Crit Care 2020; 24: 478.

3. Koeze J, Keus F, Dieperink W, van der Horst IC, Zijlstra JG, van Meurs $\mathrm{M}$. Incidence, timing and outcome of AKI in critically ill patients varies with the definition used and the addition of urine output criteria. BMC Nephrol 2017; 18: 70.

4. Wlodzimirow KA, Abu-Hanna A, Slabbekoorn M, Chamuleau RA, Schultz MJ, Bouman CS. A comparison of RIFLE with and without urine output criteria for acute kidney injury in critically ill patients. Crit Care 2012; 16: R200.

5. Macedo E, Malhotra R, Claure-Del Granado R, Fedullo P, Mehta RL. Defining urine output criterion for acute kidney injury in critically ill patients. Nephrol Dial Transplant 2011; 26: 509-15.

How to cite this article: Martins CB, De Bels D, Honore PM, Redant S. Early prediction of acute kidney injury by machine learning: Should we add the urine output criterion to improve this new tool? J Transl Intern Med 2020; 8: 201-2. 